\title{
Integral equation method for evaluation of eddy-current impedance of a tilted, surface-breaking crack
}

\author{
Lars Larsson, ${ }^{1}$ Anders Boström, ${ }^{2}$ Peter Bövik, ${ }^{2}$ and Håkan Wirdelius ${ }^{1}$ \\ ${ }^{1}$ Department of Materials and Manufacturing Technology, Chalmers University of Technology, \\ Göteborg, Sweden \\ ${ }^{2}$ Department of Applied Mechanics, Chalmers University of Technology, Göteborg, Sweden
}

(Received 26 March 2013; accepted 5 November 2013; published online 20 November 2013)

\begin{abstract}
An integral equation method for solving the eddy-current nondestructive evaluation problem for a flat, tilted, and surface-breaking crack in a conducting half-space is presented. The method involves use of a half-space Green's tensor and the Bowler potential. This potential describes the jump in the electric field over the crack and is expanded in basis functions related to the Chebyshev polynomials, being a more analytical approach than the commonly used boundary element method. In the method, the scatterer defines a transformation operator to be applied on the incoming field. This is practical in simulations of the eddy-current inspection where this operator is independent of the position of the probe. The numerical calculations of the change in impedance due to the crack are compared to a Finite Element model of the problem and good agreement is found. (C) 2013 AIP Publishing LLC. [http://dx.doi.org/10.1063/1.4832330]
\end{abstract}

\section{INTRODUCTION}

Much of the development of more and more advanced nondestructive evaluation (NDE) technologies can be traced to the introduction of structural design and risk based inspection programs based on the damage tolerance concept. New and stronger demands on reliability of used nondestructive methods and procedures have enforced different strategies to quantify the inspection capability. The most dominant and frequently used method within the aero industry is the probability of detection (POD) methodology, see, e.g., Jensen et al., ${ }^{1}$ Wirdelius and Persson, ${ }^{2}$ and Rosell and Persson. ${ }^{3}$ The intention is that POD curves should provide a statistically sound measurement of a method's capacity to detect a defect as function of its size. These POD curves can then be used to find the optimal NDE technique with respect to a specific object, material, defect size, and other defect characteristics. The proposed qualification procedure with test pieces is very expensive and it also tends to introduce a number of possible misalignments between the actual testing situation and the proposed experimental simulation. Experimentally validated simulation tools that capture variations of both the NDE methods and well-defined procedures and samples can then be used to predict the outcome of a single NDE situation and provide cost effective synthetic POD data.

Eddy-current NDE involves the detection of electromagnetic field anomalies caused by inhomogeneities in an electrically conducting material. The primary eddy-current field is usually produced by sinusoidal excitation of a small induction coil located near the surface of the inspected part. By scanning the coil over the surface, flaws can be detected by observing a change in the coil impedance due to flawinduced perturbations of eddy-current density. A mathematical model of the NDE problem is often very useful, e.g., one can obtain a better understanding of the physical process of the NDE situation and it is easier to perform parametric studies. Computer simulations of the flaw detection process require calculation of the electromagnetic field in the material around the flaw and the response from the defect, for this a reciprocity relation exists where the flaw response function can be expressed as an integral over the surface of the flaw. There have been several studies of simulating scattering of eddy-currents from cracks in a conducting half-space during recent decades, see Auld and Moulder. ${ }^{4}$ Harfield and Bowler ${ }^{5}$ give a closed form expression for the change in electromagnetic impedance of a conductor due to the presence of an infinitely long, perpendicular surface-breaking crack in a normally incident, uniform electric field. The perturbed electromagnetic field was more accurately calculated using Wiener-Hopf technique than Auld et al. ${ }^{6}$ A similar problem is considered by Kahn et al. ${ }^{7}$ Harfield and Bowler ${ }^{8}$ develop a method based on geometrical theory of diffraction (GTD) for eddy-current nondestructive evaluation. Lewis et $a l .{ }^{9}$ investigate thin skin electromagnetic scattering of a semi-elliptical surface-breaking crack in an infinite halfplane interrogated by a uniform surface current. Bowler et $a l .{ }^{10}$ calculate eddy-current probe responses for surface cracks with a finite opening. Eddy-current interaction with an ideal crack with negligible opening and acting as an impenetrable barrier to electric current is considered by Bowler. $^{11}$ The method of Bowler ${ }^{11}$ was extended by Beltrame and Burais ${ }^{12}$ to consider also the inclination of the crack.

In the present paper the mathematical modeling of a surface-breaking, infinite crack in a half-space, using an integral equation technique, is studied. The crack can have any depth and tilt. The probe is modeled as a three-dimensional single coil with a lift-off giving a three-dimensional incoming field (Dodd and Deeds ${ }^{13}$ ) towards the crack. The integral equation of Bowler ${ }^{11}$ is used. However, the solution procedure is different and instead similar to the one used by Bövik and Boström ${ }^{14}$ and Jansson and Boström ${ }^{15}$ for scattering of ultrasonic waves from internal and subsurface cracks in an 
elastic half-space. The integral equation for the jump in the tangential electrical field over the crack is solved by expanding the Green's tensor in plane waves, where the free part is expanded in the crack coordinate system and the additional part due to interface between the half-spaces is expanded in the half-space coordinate system. To discretize the integral equation an expansion of the jump in the electrical field in Chebyshev functions is performed in order to get the correct singularity at the crack edge and mouth. This gives a more analytical method which can be expected to be more numerically effective.

\section{PROBLEM STATEMENT}

Consider the typical eddy-current testing situation as depicted in Fig. 1. An eddy-current probe in the shape of a small cylindrical coil is scanning closely over the surface $z_{0}=0$, which divides space into an air-filled half-space $z_{0}<0$ and a nonferromagnetic, conducting (metal) half-space $z_{0}>0$. The properties and modeling of the probe is described in Sec. IV. The metal half-space contains an infinitely long (in the $y$ direction) surface-breaking crack of height $a$ (in the $z$ direction), which is tilted by the angle $\psi$ from the normal to the interface. The two coordinate systems

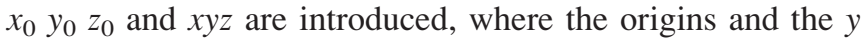
and $y_{0}$ axes along the crack mouth are coinciding. The $x_{0} y_{0}$ $z_{0}$ system has the $z_{0}$ axis normal to the interface and the $x_{0}$ axis in the interface perpendicular to the crack mouth. The $x y z$ system is tilted the angle $\psi$ around the $y_{0}$ axis so that the $z$ axis lies in the plane of the crack. The half-space $z_{0}>0$ is denoted region $i=0$ and $z_{0}<0$ as region $i=1$. The geometry of the problem is thus $2 \mathrm{D}$ (with translational invariance in the $y$ direction), but the field distribution is $3 \mathrm{D}$ because of the exciting probe.

Only time harmonic conditions are considered and the time factor $\exp (-\mathrm{i} \omega t)$ is suppressed throughout. The wave numbers in the two regions are $k_{i}^{2}=\mathrm{i} \mu_{i} \omega \sigma_{i}+\omega^{2} \mu_{i} \varepsilon_{i}$, where $\mu_{i}$ is the magnetic permeability, $\varepsilon_{i}$ the electric permittivity, and $\sigma_{i}$ is the conductivity, for $i=0,1$. In the air the constants are taken as those of vacuum, so the conductivity is vanishing. In realistic eddy-current applications the wavelengths in air are very much larger than all relevant geometrical lengths so the wave number is practically zero. However, it is retained as nonzero here for the sake of generality. The electromagnetic fields satisfy Maxwell's equations, but after eliminations this gives for the electric field $\boldsymbol{E}_{i}$

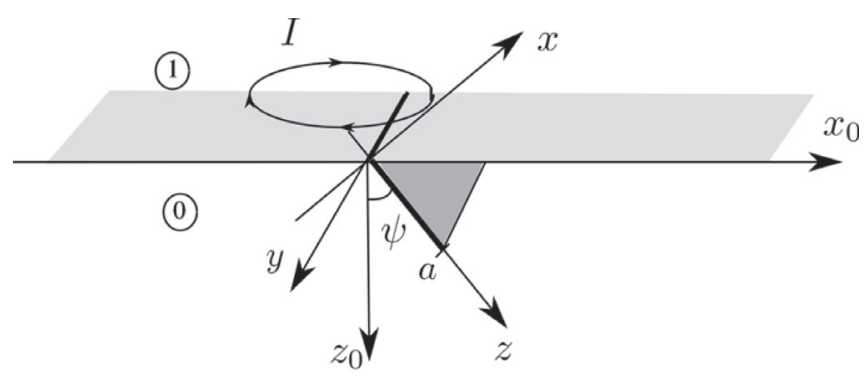

FIG. 1. The 3D scattering geometry with the metal half-space $z_{0}>0$, the air half-space $z_{0}<0$, the coil, and the tilted crack along the $z$ axis.

$$
\nabla \times \nabla \times \boldsymbol{E}_{i}-k_{i}^{2} \boldsymbol{E}_{i}=0, \quad i=0,1 .
$$

Between the two half-spaces the tangential parts of the electric and magnetic fields must be continuous

$$
\begin{aligned}
\hat{z}_{0} \times \boldsymbol{E}_{0} & =\hat{z}_{0} \times \boldsymbol{E}_{1}, \\
\frac{1}{\mu_{0}} \hat{z}_{0} \times\left(\nabla \times \boldsymbol{E}_{0}\right) & =\frac{1}{\mu_{1}} \hat{z}_{0} \times\left(\nabla \times \boldsymbol{E}_{1}\right),
\end{aligned}
$$

where $\hat{z}_{0}$ is the unit vector in the $z_{0}$ direction. The crack is assumed to be infinitely thin but with limited electric contact between the faces so the boundary conditions across the crack are taken as

$$
\begin{gathered}
\hat{x} \times\left(\nabla \times \boldsymbol{E}_{0}^{-}\right)=\hat{x} \times\left(\nabla \times \boldsymbol{E}_{0}^{+}\right), \\
E_{0 x}^{-}=E_{0 x}^{+}=\alpha V,
\end{gathered}
$$

where the indices plus and minus denote the limit from the two sides $x>0$ and $x<0$, respectively. Here, $V$ is a scalar surface field on the crack that determines the jump in the tangential electric field and $\alpha$ is a constant which determines the degree of contact across the crack. For an open, infinitely thin crack $\alpha=0$. To model a crack with partial contact the following model proposed by Harfield and Bowler ${ }^{16}$ is used:

$$
\alpha=\frac{\sigma_{f}}{c\left(\sigma_{f}-\sigma_{0}\right)} .
$$

This results in an electric contact corresponding to the case of a volumetric flaw of the width $c$ and the electric conductivity $\sigma_{f}$. To make the problem formulation complete, the electric field must also satisfy radiation conditions.

\section{INTEGRAL EQUATION SOLUTION}

To solve the scattering problem the integral equation derived by Bowler ${ }^{11}$ is used, but with a nonzero right-hand side if electrical contact exists across the crack. This equation contains the Green's tensor for the two half-spaces without the crack, and the equation therefore only contains an integral over the crack

$$
\begin{aligned}
& E_{0 x}^{\text {inc }}(0, y, z)+k_{0}^{2} \lim _{x \rightarrow 0^{+}} \int_{-\infty}^{\infty} \int_{0}^{a} G_{11}\left(x, y, z ; 0, y^{\prime}, z^{\prime}\right) \\
& \quad \times V\left(y^{\prime}, z^{\prime}\right) \mathrm{d} z^{\prime} \mathrm{d} y^{\prime}=E_{0 x}^{+}(0, y, z),
\end{aligned}
$$

where $E_{0 x}^{\text {inc }}(0, y, z)$ is the incoming field, i.e., the field from the coil in the absence of the crack. The unknown $V$ is a surface scalar function defined on the crack such that it determines the jump in electric field through $\nabla^{\prime} V\left(y^{\prime}, z^{\prime}\right)$ $=\boldsymbol{E}_{0}^{-}\left(0, y^{\prime}, z^{\prime}\right)-\boldsymbol{E}_{0}^{+}\left(0, y^{\prime}, z^{\prime}\right)$. Only the 11 component of the Green's tensor is needed as only the normal $(x)$ component of the integral representation is used and the unknown only has a normal component. ${ }^{11}$ According to the boundary condition (5) the right-hand side of Eq. (7) can be written as $\alpha V(y, z)$. It is observed that the resulting integral equation is singular, but the way of solving it makes it possible to later take the limit under the integral. 
The Green's tensor is divided into two parts, where one is the free-space tensor of the metal and the other is due to the interface to the air. The free-space Green's tensor is obtained by taking a double Fourier transform in the tangential coordinates $y_{0}$ and $z_{0}$. This straightforwardly gives

$$
\begin{aligned}
& G_{n n^{\prime}}^{\mathrm{free}}\left(x, y, z ; x^{\prime}, y^{\prime}, z^{\prime}\right) \\
& \quad=2 \mathrm{i} \sum_{j} \int_{-\infty}^{\infty} \int_{-\infty}^{\infty} \frac{\mathrm{d} q \mathrm{~d} p}{h} f_{j n^{\prime}} f_{j n}^{*} \mathrm{e}^{\mathrm{i}\left(h\left|z^{\prime} 0-z_{0}\right|+p\left(y_{0}^{\prime}-y_{0}\right)+q\left(x_{0}^{\prime}-x_{0}\right)\right)},
\end{aligned}
$$

where $j$ is summed over 1,2 and

$$
\begin{aligned}
& f_{11}=0, \quad f_{12}=\frac{\mathrm{i} k_{0} q}{4 \pi k_{0} s}, \quad f_{13}=\frac{-\mathrm{i} k_{0} p}{4 \pi k_{0} s}, \\
& f_{21}=\frac{s}{4 \pi k_{0}}, \quad f_{22}=\frac{-p h}{4 \pi k_{0} s}, \quad f_{23}=\frac{-q h}{4 \pi k_{0} s} .
\end{aligned}
$$

Here, $q$ and $p$ are Fourier transform variables in $x_{0}$ and $y_{0}$, respectively, and $h=\sqrt{k_{0}^{2}-s^{2}}$ and $s^{2}=p^{2}+q^{2}$ (the square root is defined such that $\Im(\sqrt{(*)}) \geq 0$ ). Furthermore, $f^{*}$ is obtained from $f$ by changing all explicit $\mathrm{i}$ to $-\mathrm{i}$. It is noted that this expansion of the Green's tensor can be viewed as an expansion in plane waves. The free space Green's tensor is written in the coordinate system $x_{0} y_{0} z_{0}$ of the interface, but this part has of course the same appearance in all coordinate systems, in particular, also in the crack system $x y z$.

To satisfy the boundary conditions at the metal-air interface an additional part is added to the Green's tensor, in the metal this is a reflected part and in the air a transmitted one. As the Green's tensor is just an expansion in plane waves, the appearing reflection and transmission coefficients are just the well-known ones for plane waves. Using the boundary conditions (2) and (3) to determine the reflection and transmission coefficients and then transforming to the crack system the reflected part in the metal becomes

$G_{n n \prime}^{\mathrm{refl}}\left(x, y, z ; x^{\prime}, y^{\prime}, z^{\prime}\right)=2 \mathrm{i} \sum_{j j^{\prime}} \int_{-\infty}^{\infty} \int_{-\infty}^{\infty} \frac{\mathrm{d} q \mathrm{~d} p}{h} g_{j n^{\prime}} R_{j j^{\prime}} g_{j \prime n}^{+} \cdot \mathrm{e}^{\mathrm{i}(x \prime(q \cos \psi-h \sin \psi)-x(q \cos \psi+h \sin \psi)+p(y \prime-y)+z \prime(h \cos \psi+q \sin \psi)+z(h \cos \psi-q \sin \psi))}$,

where $j$ and $j^{\prime}$ are summed over 1,2 and

$$
\begin{aligned}
& g_{11}=\frac{\mathrm{i} p \cos \psi}{4 \pi s}, \quad g_{12}=\frac{-\mathrm{i} q}{4 \pi s}, \quad g_{13}=\frac{\mathrm{i} p \sin \psi}{4 \pi s}, \\
& g_{21}=\frac{-q h \cos \psi-s^{2} \sin \psi}{4 \pi k_{0} s}, \quad g_{22}=\frac{-p h}{4 \pi k_{0} s}, \\
& g_{23}=\frac{s^{2} \cos \psi-q h \sin \psi}{4 \pi k_{0} s} .
\end{aligned}
$$

Here, $g^{+}$is obtained from $g$ by changing $\mathrm{i}$ to $-\mathrm{i}$ and $h$ to $-h$. The standard Fresnel reflection coefficients are

$$
\begin{aligned}
& R_{11}=\frac{\sqrt{k_{0}^{2}-s^{2}}-b_{01} \sqrt{k_{1}^{2}-s^{2}}}{\sqrt{k_{0}^{2}-s^{2}}+b_{01} \sqrt{k_{1}^{2}-s^{2}}}, \\
& R_{22}=\frac{b_{01} k_{1}^{2} \sqrt{k_{0}^{2}-s^{2}}-k_{0}^{2} \sqrt{k_{1}^{2}-s^{2}}}{b_{01} k_{1}^{2} \sqrt{k_{0}^{2}-s^{2}}+k_{0}^{2} \sqrt{k_{1}^{2}-s^{2}}},
\end{aligned}
$$

where $b_{01}=\mu_{0} / \mu_{1}$ and the two off-diagonal elements are of course zero: $R_{12}=R_{21}=0$.

To discretize the integral equation and to take care of the hypersingularity, the unknown $V\left(y^{\prime}, z^{\prime}\right)$ is expanded as

$$
V\left(y^{\prime}, z^{\prime}\right)=\sum_{m^{\prime}} \int_{-\infty}^{\infty} \beta_{m^{\prime}}\left(p^{\prime}\right) \phi_{m^{\prime}}\left(z^{\prime}\right) e^{-i p / y \prime} \mathrm{d} p^{\prime}
$$

where $\beta_{m^{\prime}}\left(p^{\prime}\right)$ are the unknown expansion coefficients. Here, the expansion functions are defined as

$$
\phi_{m^{\prime}}\left(z^{\prime}\right)=\cos \left(\left(2 m^{\prime}-1\right) \arcsin \left(z^{\prime} / a\right)\right), \quad m^{\prime}=1,2,3, \ldots,
$$

and the summation is over all positive integers. By expanding $V\left(y^{\prime}, z^{\prime}\right)$ in these functions, which are related to the Chebyshev polynomials, the correct weak singularity appears at the crack edge, see Bowler. ${ }^{11}$ At the crack mouth the expansion functions have a finite value to admit a finite value of the jump in electric field. Inserting the sum of the expansions of the Green's tensor (8) and (10) and the expansion of the unknown (13) into the integral Eq. (7), projecting the result on the Chebyshev functions and taking a Fourier transform in $y$ yields

$$
\sum_{m^{\prime}} \beta_{m^{\prime}}(p) Q_{m^{\prime} m}(p)=-\int_{0}^{a} \int_{-\infty}^{\infty} E_{0 x}^{\mathrm{inc}}(0, y, z) \mathrm{e}^{\mathrm{i} p y} \phi_{m}(z) \mathrm{d} y \mathrm{~d} z
$$

where

$$
\begin{aligned}
Q_{m^{\prime} m}(p)= & \frac{\mathrm{i} a^{2}}{2} \int_{-\infty}^{\infty} \frac{\mathrm{d} q}{h}\left(s^{2} I_{m}(q a) I_{m^{\prime}}(-q a)\right. \\
& +\left(\frac{\cos ^{2} \psi}{s^{2}}\left(k_{0}^{2} p^{2} R_{11}-q^{2} h^{2} R_{22}\right)\right. \\
& \left.+\left(s^{2} \sin ^{2} \psi\right) R_{22}\right) I_{m}(h a \cos \psi-q a \sin \psi) \\
& \left.\times I_{m^{\prime}}(h a \cos \psi+q a \sin \psi)\right) \\
& -2 \pi \alpha \int_{0}^{a} \phi_{m^{\prime}}(z) \phi_{m}(z) \mathrm{d} z .
\end{aligned}
$$

Here, the following function is introduced:

$$
I_{m}(\gamma)=\int_{0}^{1} \cos ((2 m-1) \arcsin t) \mathrm{e}^{\mathrm{i} \gamma t} \mathrm{~d} t .
$$


It seems that this integral can not be calculated in simple analytical form, but except for large arguments it is straightforward to compute numerically. For an interior crack, with a slightly different expansion of the unknown, the corresponding integral is essentially a Bessel function. ${ }^{14}$ It should be noted that the last term in expression (16) can be calculated analytically.

\section{THE PROBE}

The eddy-current probe is assumed to be a coil with axis perpendicular to the interface between the air and the metal. An analytical expression for the magnetic vector potential is given in Dodd and Deeds. ${ }^{13}$ By use of this expression the electric field in the metal in the absence of the crack is written as

$$
\begin{aligned}
\boldsymbol{E}_{0}^{\mathrm{inc}}\left(x_{0}, y_{0}, z_{0}\right)= & -\frac{\omega \mu_{0} J}{2 \pi} \int_{-\infty}^{\infty} \int_{-\infty}^{\infty} \frac{1}{s^{2}} Y\left(r_{2}, r_{1}\right) \mathrm{e}^{\mathrm{i}\left(q x_{0}+p y_{0}+h z_{0}\right)} \\
& \times\left(\mathrm{e}^{-s l_{1}}-\mathrm{e}^{-s l_{2}}\right) \frac{(p,-q, 0)}{s-i h} \mathrm{~d} p \mathrm{~d} q
\end{aligned}
$$

where $l_{1}$ is the lift-off, $l_{2}-l_{1}$ the height of the probe, and $J$ is the current density, assumed to be constant inside the coil. The function $Y\left(r_{2}, r_{1}\right)$ is an integration in $r$ over the radial extension of the probe

$$
Y\left(r_{2}, r_{1}\right)=\int_{r_{1}}^{r_{2}} r J_{1}(r s) \mathrm{d} r
$$

where, $r_{1}$ and $r_{2}$ are the inner and outer radii of the coil, respectively, and $J_{1}(r s)$ is a Bessel function. Inserting the expression for the incoming field in Eq. (15) and carrying out the integrations over $y$ and $p$ the right-hand side of the equation can be written

$$
\begin{aligned}
& -\int_{0}^{a} \int_{-\infty}^{\infty} E_{0 x}^{\mathrm{inc}}(0, y, z) \mathrm{e}^{\mathrm{i} p y} \phi_{m}(z) \mathrm{d} y \mathrm{~d} z \\
& =-\omega \mu_{0} a J \int_{-\infty}^{\infty} \frac{p \cos \psi}{s^{2}(s-\mathrm{i} h)} Y\left(r_{2}, r_{1}\right)\left(\mathrm{e}^{-s l_{1}}-\mathrm{e}^{-s l_{2}}\right) \\
& \quad \times I_{m}(h a \cos \psi+q a \sin \psi) \mathrm{d} q .
\end{aligned}
$$

The remaining integral has to be computed numerically.

To calculate the eddy-current probe impedance change $\Delta Z$ due to the crack it is convenient to use the following expression (see Bowler et al. ${ }^{10}$ ):

$$
\Delta Z=\frac{\sigma_{f}-\sigma_{0}}{I^{2}} \int_{0}^{a} \int_{-\infty}^{\infty} E_{0 x}^{\mathrm{inc}}(\boldsymbol{r}) V(y, z) \mathrm{d} y \mathrm{~d} z,
$$

where $I$ is the total current in the coil when acting as a transmitter. Using Eq. (18) together with the expansion (13) of $V(y, z)$ yields

$$
\begin{aligned}
\Delta Z= & \frac{\left(\sigma_{0}-\sigma_{f}\right) \omega \mu_{0} a J}{k_{0}^{2} I^{2}} \sum_{m} \int_{-\infty}^{\infty} \int_{-\infty}^{\infty} \beta_{m}(p) \frac{p \cos \psi}{s^{2}(s-\mathrm{i} h)} \\
& \times Y\left(r_{2}, r_{1}\right)\left(\mathrm{e}^{-s l_{1}}-\mathrm{e}^{-s l_{2}}\right) \cdot I_{m}(h a \cos \psi+q a \sin \psi) \mathrm{d} q \mathrm{~d} p .
\end{aligned}
$$

The computation of this quantity is now reduced to quadratures and the solution of the system of Eq. (15). It is noted that this system of equations has to be solved for each value of $p$ needed to compute the $p$ integral in the impedance change (22).

\section{NUMERICAL EXAMPLES}

In this section numerical examples are presented and compared with a FEM solution. The implementation is rather straightforward and is performed in MATLAB. First, this involves the numerical integration in (16) and (20). This is straightforward except that the integral in (16) has to be handled with care as each term individually is divergent. So the integral must be computed as a whole and to get reasonable convergence the dominate behaviour for large arguments is calculated and integrated analytically. Solving the system of Eq. (15), there only remains to compute the integral in Eq. (22). Here, the $q$ integral is straightforward. It is noted that the $p$ integral in Eq. (22) involves a recomputation of all the previous steps. This reflects the fact that the problem is in effect solved by a Fourier transform in $y$ (with transform variable $p$ ), which results in a $2 \mathrm{D}$ problem for each $p$. comsol Multiphysics is used to obtain the FEM solution. In the used FEM model (Rosell and Persson ${ }^{17}$ ) the crack must have a finite width (in the $x$ direction) and this has a clear influence on the results, see Rosell and Persson, ${ }^{17}$ where the effect of crack width is investigated. In the model contact across the crack is modelled by a finite conductivity, resulting in a volumetric flaw of width $c$ and conductivity $\sigma_{f}$. Obviously, the FEM mesh and the crack length (in the $y$ direction) are finite and the crack and the FEM mesh are in the present case $7 \mathrm{~mm}$ long, which is long enough to simulate an infinite crack and an infinite region with reasonable accuracy.

The material is titanium with the conductivity $\sigma=0.58 \mathrm{MS} / \mathrm{m}$ and $\mu$ and $\epsilon$ are the same as those of vacuum. The probe is a single coil with height $1 \mathrm{~mm}$, inner diameter $0.75 \mathrm{~mm}$, outer diameter $1 \mathrm{~mm}$, and lift-off $0.1 \mathrm{~mm}$. There is no electric contact across the crack $(\alpha=0)$ unless stated otherwise. The change in impedance $\Delta Z$ during a scan perpendicular over the crack is presented for a few cases below.

Figure 2 shows a comparison between the present method and FEM for the frequency $1 \mathrm{MHz}$. The crack height (along the $z$ axis) is chosen as $0.66 \mathrm{~mm}$, corresponding to one skin depth at this frequency. This choice is critical in the sense that there is a field varying with depth which is reasonably large also at the crack tip. Two different crack tilts are shown and two FEM solutions are given for the crack widths $50 \mu \mathrm{m}$ and $100 \mu \mathrm{m}$. If the two FEM results are extrapolated, it is seen that the results agree very well with the present solution. That a wider crack gives a larger change in impedance is in agreement with the results in Refs. 17 and 18.

To illustrate the effect of electric contact across the crack, Fig. 3 shows the real versus the imaginary part of $\Delta Z$ during a surface scan. The frequency and the height of the crack are kept the same as in Fig. 2. As expected an increased electric contact gives a smaller $\Delta Z$.

In Fig. 4, the change in impedance for four different crack tilts, but with the same depth, are shown for the 

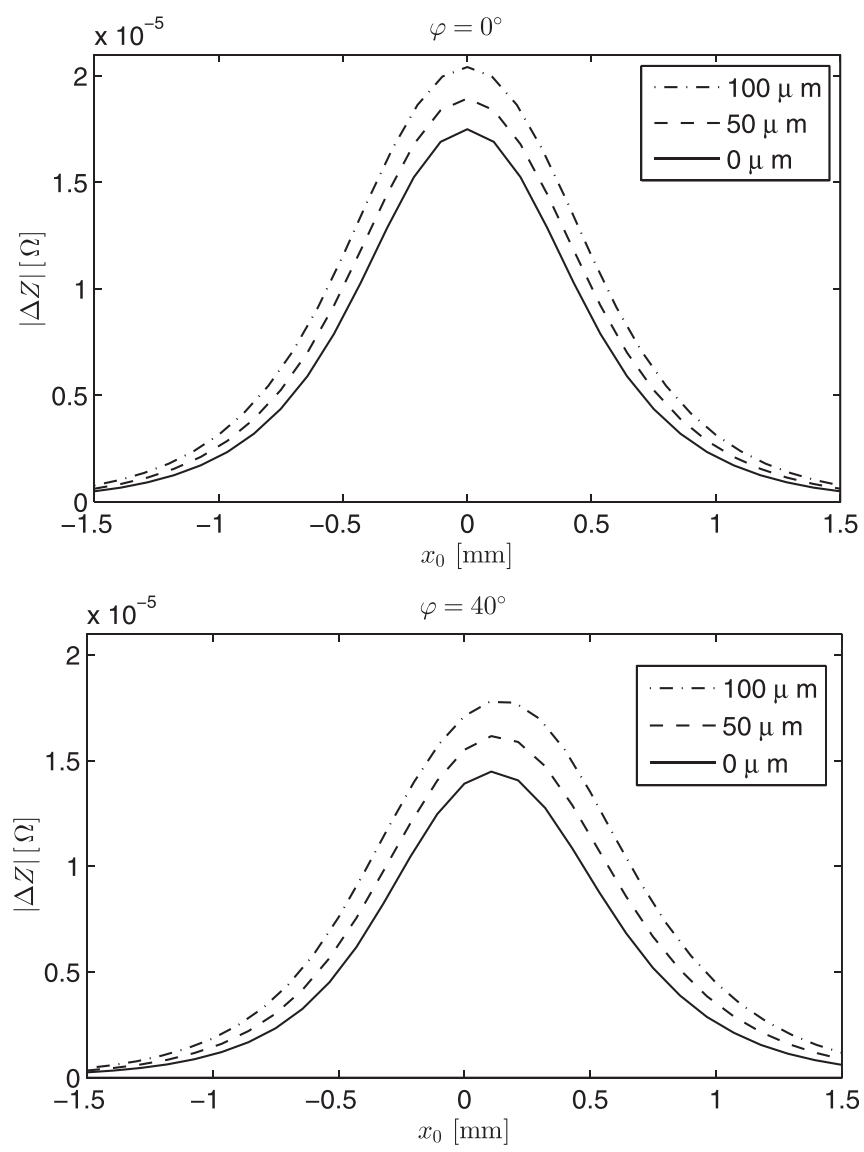

FIG. 2. The absolute value of $\Delta Z$ as a function of position during a surface scan for the tilt angles $\psi=0^{\circ}$ and $\psi=40^{\circ}$. The full-drawn curves are with the present method, the dashed with FEM and crack width $50 \mu \mathrm{m}$, and the dashed-dotted with FEM and crack width $100 \mu \mathrm{m}$.

frequency $1 \mathrm{MHz}$. The extension of the cracks in the $z_{0}$ direction (which is $a \cos \psi$ ) is $0.66 \mathrm{~mm}$ or one skin depth. This results in different crack height along the $z$ axis for different crack tilts. It seems like the width of the signal could be a

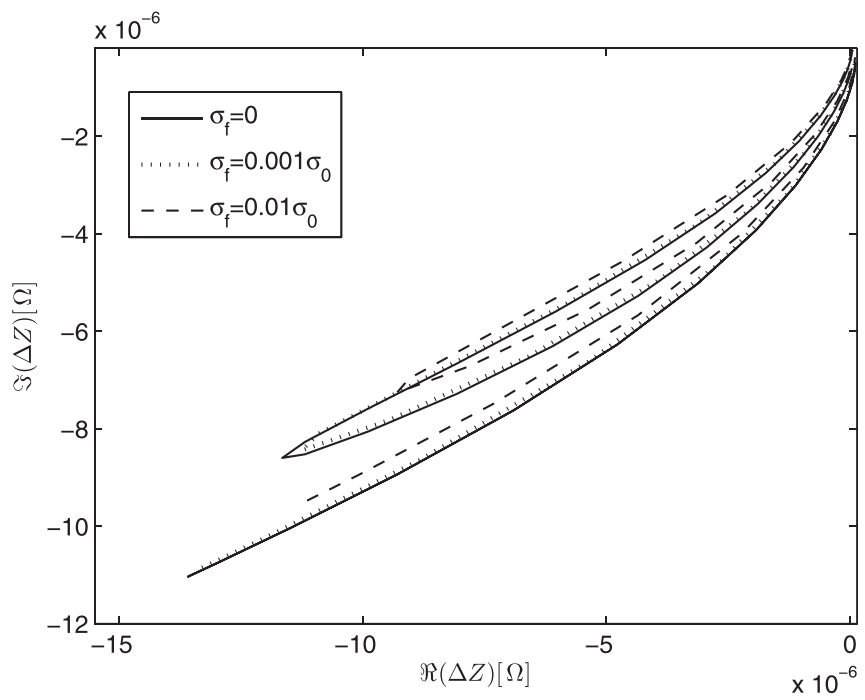

FIG. 3. The real versus the imaginary part of $\Delta Z$ during a surface scan. The tilt angles are $\psi=0^{\circ}$ and $40^{\circ}$ (starting from the bottom). The electric conductivity inside the crack $\sigma_{f}$ is zero for the solid curves, $\sigma_{f}=0.001 \cdot \sigma_{0}$ for the dotted curves, and $\sigma_{f}=0.01 \cdot \sigma_{0}$ for the dashed curves. The thickness parameter $c=50 \mu \mathrm{m}$.

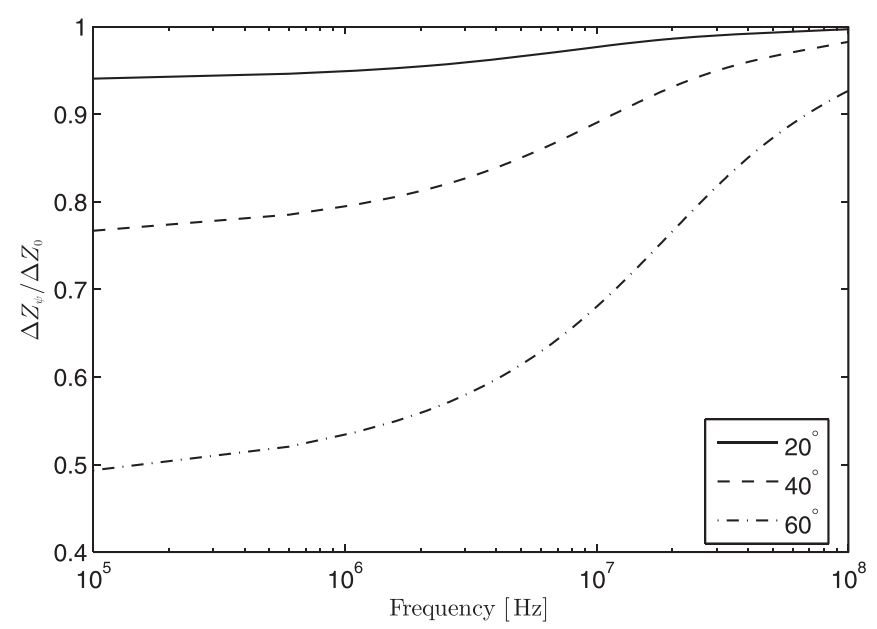

FIG. 4. The real versus the imaginary part of $\Delta Z$ during a surface scan. The tilt angles are $\psi=0^{\circ}$ for the solid curve, $20^{\circ}$ for the dashed curve, $40^{\circ}$ for the dotted curve, and $60^{\circ}$ for the dashed-dotted curve.

good measure of different tilt angles. The maximum amplitude, on the other hand, is almost the same for all of the cracks.

Figure 5 shows the change in impedance as a function of frequency for tilted defects relative the change in impedance for a defect normal to the surface. For all frequencies the coil is centered above the crack mouth, which is near the position where maximum amplitude is achieved. The height of the cracks are $0.66 \mathrm{~mm}$ and the range of frequency is chosen as $10^{5}-10^{8} \mathrm{~Hz}$, which is a typical frequency range used for titanium in the aero industry. The comparison shows that the maximum amplitude, which is mostly dependent on the depth of the crack, can be used to distinguish different tilt angles if the frequency is not too high. In the thin skin frequency domain where the amplitude is almost independent of the depth of the crack the difference in amplitude between different tilt angles is very small.

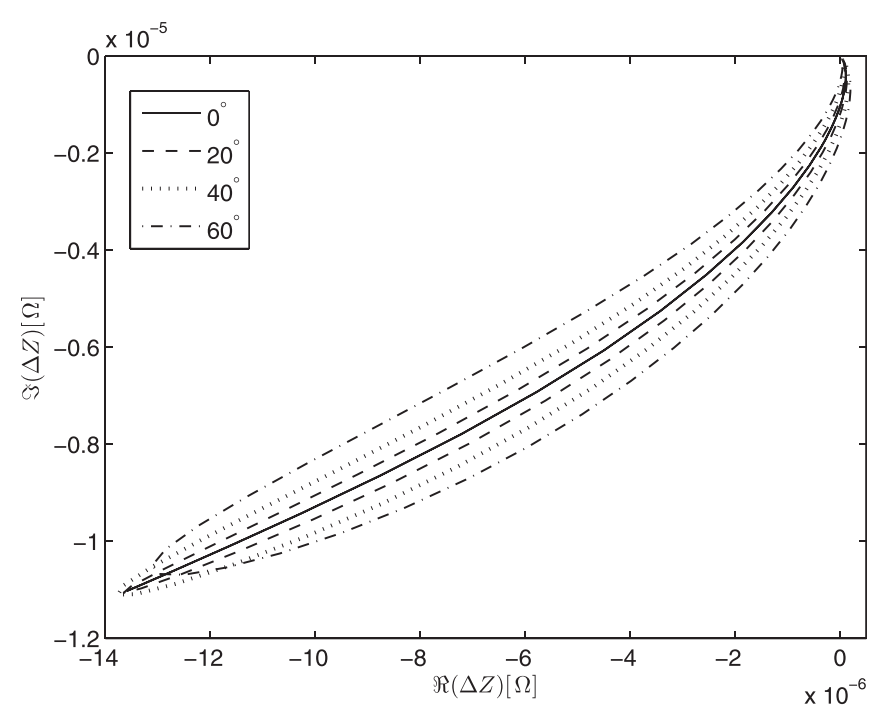

FIG. 5. The maximum amplitude of the change in impedance for a tilted crack relative the maximum amplitude of the change in impedance for a crack with no tilt, plotted against the frequency. Tilt angles are $20^{\circ}, 40^{\circ}$, and $60^{\circ}$, starting from the top. 

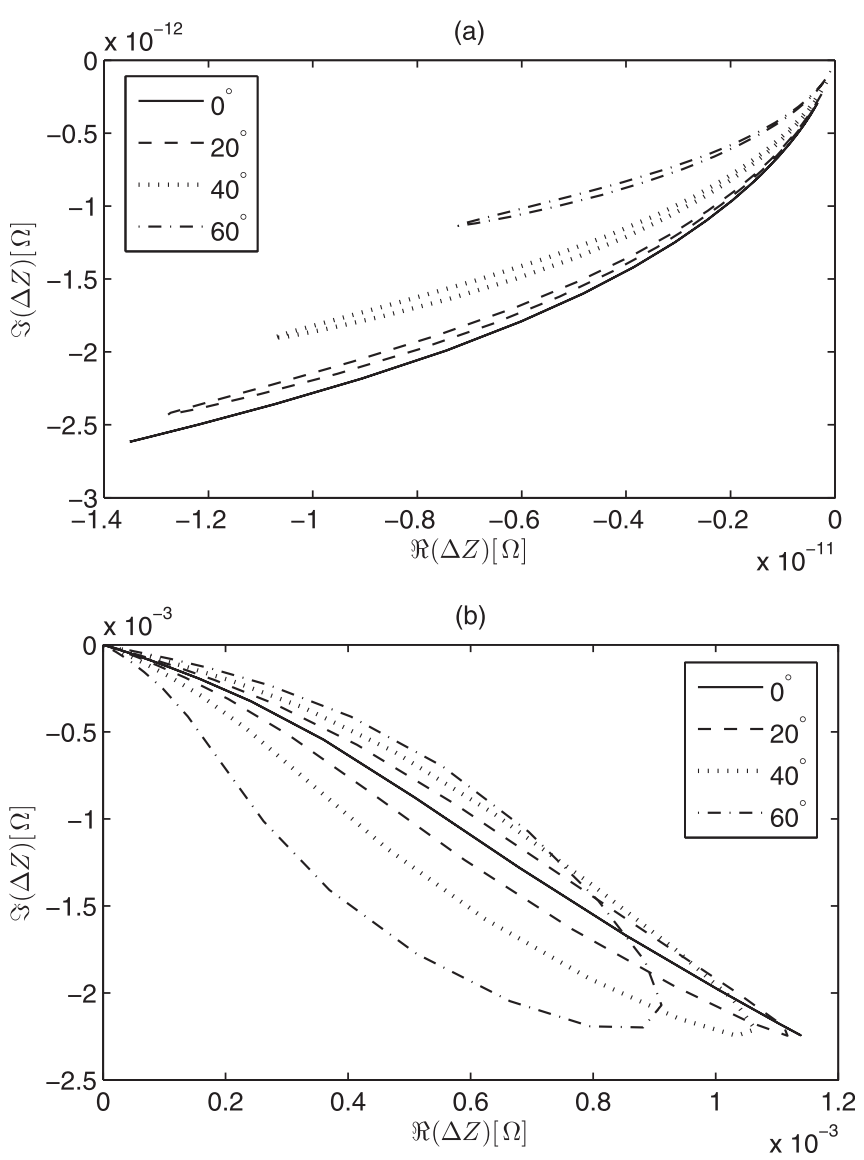

FIG. 6. The real versus the imaginary part of $\Delta Z$ during a surface scan for the frequency $10^{5} \mathrm{~Hz}$ in (a) and $10^{8} \mathrm{~Hz}$ in (b). The tilt angles are $\psi=0^{\circ}$ for the solid curve, $20^{\circ}$ for the dashed curve, $40^{\circ}$ for the dotted curve, and $60^{\circ}$ for the dashed-dotted curve.

To explore the frequency dependence further, Figs. 6(a) and $6(\mathrm{~b})$ present the change in impedance during a surface scan for a low $\left(10^{5} \mathrm{~Hz}\right)$ and a high $\left(10^{8} \mathrm{~Hz}\right)$ frequency, respectively. The crack height (along the $z$ axis) is chosen as $0.66 \mathrm{~mm}$. Again there is a clear distinction between the different tilt angles, for the low frequency in amplitude and for the high frequency in the width of the signals. If the tilt angle is to be measured a higher frequency seems to be favourable, whereas the difference in depth as might have been expected is more evident when the frequency is not too high. It is noted that there is a change in sign in the real part between Figs. 6(a) and 6(b). The large change in amplitude for different frequencies is a matter of the chosen normalization, which has kept the current amplitude the same irrespective of the frequency, thus resulting in more input effect at higher frequencies. In a real experiment it is rather the effect that is kept constant.

\section{CONCLUDING REMARKS}

The eddy-current interaction problem for a tilted, flat, surface-breaking crack is solved using an integral equation method. The jump in the electric field over the crack (known as the Bowler potential) is expanded in basis functions related to the Chebyshev polynomials. The transformation operator is generated and by reciprocity used to calculate the change in impedance due to the crack. These calculations show good agreement with a FEM solution of the problem. The crack in the present paper is infinite, but the method can be modified to handle finite cracks. The method is applicable to other surface geometries than a plane and another possible option is to add an extra layer above the crack.

\section{ACKNOWLEDGMENTS}

The authors wish to thank Anders Rosell for carrying out the FEM calculations. The work in the present paper is a part of the European project "PICASSO" and the funding from the European Commission within the FP7 programme is gratefully acknowledged.

${ }^{1}$ F. Jensen, S. Mahaut, P. Calmon, and C. Poidevin, "Simulation based POD evaluation of NDI techniques," in Proceedings of 10th European Conference on Nondestructive Testing, Moscow, 2010.

${ }^{2} \mathrm{H}$. Wirdelius and G. Persson, "Simulation based validation of the detection capacity of an ultrasonic inspection procedure," Int. J. Fract. 41, 23-29 (2012).

${ }^{3}$ A. Rosell and G. Persson, "Model based capability assessment of an automated eddy current inspection procedure on flat surfaces," Res. Nondestruct. Eval. 24, 154-176 (2013).

${ }^{4}$ B. A. Auld and J. C. Moulder, "Review of advances in quantitative eddy current nondestructive evaluation," J. Nondestruct. Eval. 18, 3-36 (1999).

${ }^{5} \mathrm{~N}$. Harfield and J. R. Bowler, "Analysis of eddy-current interaction with a surface-breaking crack," J. Appl. Phys. 76, 4853-4856 (1994).

${ }^{6}$ B. A. Auld, F. Muennemann, and D. K. Winslow, "Eddy current probe response to open and closed surface flaws," J. Nondestruct. Eval. 2, 1-21 (1981).

${ }^{7}$ A. H. Kahn, R. Spal, and A. Feldman, "Eddy-current losses due to a surface crack in conducting material," J. Appl. Phys. 48, 4454-4459 (1977).

${ }^{8}$ N. Harfield and J. R. Bowler, "A geometrical theory for eddy-current nondestructive evaluation," Proc. R. Soc. London, Ser. A 453, 1121-1152 (1997).

${ }^{9}$ A. M. Lewis, D. H. Michael, M. C. Lugg, and R. Collins, "Thin-skin electromagnetic fields around surface-breaking cracks in metals," J. Appl. Phys. 64, 3777-3784 (1988).

${ }^{10}$ J. R. Bowler, S. A. Jenkins, L. D. Sabbagh, and H. A. Sabbagh, "Eddy-current probe impedance due to a volumetric flaw," J. Appl. Phys. 70, 1107-1114 (1991).

${ }^{11}$ J. R. Bowler, "Eddy-current interaction with an ideal crack. I. The forward problem," J. Appl. Phys. 75, 8128-8137 (1994).

${ }^{12} \mathrm{P}$. Beltrame and N. Burais, "Generalization of the ideal crack model in eddy-current testing," IEEE Trans. Magn. 40, 1366-1369 (2004).

${ }^{13}$ C. V. Dodd and W. E. Deeds, "Analytical solutions to eddy-current probecoil problems," J. Appl. Phys. 39, 2829-2838 (1968).

${ }^{14} \mathrm{P}$. Bövik and A. Boström, "A model of ultrasonic nondestructive testing for internal and subsurface cracks," J. Acoust. Soc. Am. 102, 2723-2733 (1997).

${ }^{15} \mathrm{P}$.- $\AA$. Jansson and A. Boström, "Modeling of ultrasonic nondestructive testing of surface-breaking cracks," in Proceedings of 18th World Conference on Nondestructive Testing, Durban, South Africa, 2012.

${ }^{16} \mathrm{~N}$. Harfield and J. R. Bowler, "A thin skin theory of current leakage across surface cracks," in Electromagnetic Nondestructive Evaluation(II), edited by N. Albanese, G. Rubinacci, T. Takagi, and S. Udpa (IOS Press, 1998).

${ }^{17}$ A. Rosell and G. Persson, "Finite element modelling of closed cracks in eddy current testing," Int. J. Fatigue 41, 30-38 (2012).

${ }^{18}$ R. E. Beissner, "Slots vs. cracks in eddy current NDE," J. Nondestruct. Eval. 13, 175-183 (1994). 